\title{
Statistical Study of Admitted Typhoid Fever Patients at Bishop Shanahan Hospital, Nsukka, Enugu State
}

\author{
Didiugwu Amarachi Favour, Arum Kingsley Chinedu*, Oranye Henrietta Ebele, \\ Didiugwu Chizoba Maryann, Agu Pascal Ifeanyi
}

Department of Statistics, Faculty of Physical Science, University of Nigeria Nsukka, Nsukka, Enugu State, Nigeria

\section{Email address:}

amarachi.didiugwu@gmail.com (D. A. Favour.), kingsley.arum@unn.edu.ng (A. K. Chinedu), henrietta.oranye@unn.edu.ng (O. H. Ebele), chizoba.maryann@yahoo.com (D. C. Maryann), agu.ifeanyi@gmail.com (A. P. Ifeanyi)

${ }^{*}$ Corresponding author

\section{To cite this article:}

Didiugwu Amarachi Favour, Arum Kingsley Chinedu, Oranye Henrietta Ebele, Didiugwu Chizoba Maryann, Agu Pascal Ifeanyi. Statistical Study of Admitted Typhoid Fever Patients at Bishop Shanahan Hospital, Nsukka, Enugu State. International Journal on Data Science and Technology. Vol. 6, No. 3, 2020, pp. 60-65. doi: 10.11648/j.ijdst.20200603.11

Received: August 6, 2020; Accepted: August 24, 2020; Published: December 11, 2020

\begin{abstract}
Typhoid fever is a bacterial infection that spread through contaminated food or water, affecting many organs in human body; it has raised a very serious health threat in some developing nation, especially in Nigeria. This work is based on the statistical study of typhoid fever patients admitted at Bishop Shanahan hospital for a period of five years from January 2012 through December 2016. The data for the study was collected from in-patients record files of the above mentioned hospital. The data was presented according to duration of stay of patients in the hospital, gender of the patients, type of typhoid fever each patient suffers from and age of the patients respectively. It was also observed from the figure that age brackets of 19-36 are prone to typhoid fever than other brackets but people who are 73 years of age and above stay longer in the hospital as a result of weak immunity due to old age. Chi-square test of independency was used to analyze the data and it was found that duration of stay of typhoid fever patients is independent of gender of the patient but is dependent on type of typhoid fever patient suffers from, age and the doctor that attended to the patients.
\end{abstract}

Keywords: Diseases, Contingency Table, Chi-Square, Goodness-of-fit

\section{Introduction}

Typhoid fever is a disease that affects most West African countries and some parts of Asia like India. Typhoid fever affects mainly underdeveloped and developing countries. Typhoid and paratyphoid fevers are vastly disseminated systematic diseases commonly known as enteric fever. In most endemic areas, approximately 90 percent of the enteric fever cases are actually typhoid fever caused by salmonella enterica serovar typhi [1]. Typhoid fever is an infection of the intestinal tract and occasionally the blood stream. Typhoid fever is a bacterial illness caused by Salmonella typhi (S-typhi), one type of the salmonella bacteria group. This highly adapted human pathogen (salmonella enterica) has remarkable mechanisms for persistence in its host that helps to ensure its survival and transmission [2]. One of the most famous carriers was Mallon Mary, a cook who infected at least 51 people in U.S.A [3].
Typhoid fever happened to be the major cause of illness in most countries. According to [4], there should be provision of clean water and good sewage systems which will lead to a dramatic decrease in the incidence of typhoid in some regions in the countries; recently the burden of the disease occurs in the developing world where sanitary conditions remain poor. Reliable data from which to estimate the burden of disease in these areas are difficult to obtain, since many hospitals do not have the facilities for blood culture and up to 90 percent of patients with typhoid are treated as outpatients. [5].

Although antibiotics have remarkably reduced the rate of typhoid fever in the developed world, it remains endemic in developing countries [6].

Anyone can get typhoid fever but the risk is greatest among travelers visiting countries where the disease is common. Occasionally, local cases can be traced to exposure to a person who is a chronic carrier. Salmonella typhi is passed in the feces and to some extent, the urine of infected 
people. The germs are spread by eating or drinking water or foods contaminated by feces from the infected individual [7]. The bacteria enter the body through the mouth to cause disease. Large epidemics are most often related to the contamination of water supplies or street vended foods.

Specific antibiotics such as ampicillin, trimethoprimsulfamethoxazole or ciprofloxacin are often used to treat cases of typhoid. A vaccine is available but is generally reserved for people travelling to underdeveloped countries where significant exposure may occur. The most effective means of prevention is strict attention to food and water precautions when travelling to such countries. Although in Nigeria, malaria and typhoid are said to be endemic, malaria is far more likely to cause fever than typhoid fever.

\subsection{Statement of Problem}

Factors that contribute to the duration of stay of a typhoid fever patient in a hospital have always been a question in people's mind. This study tries to check those factors that can affect a typhoid fever patient based on the record of data which is being kept by the hospital.

\subsection{Significance of the Study}

This study will educate people on the causes of typhoid fever, symptoms, prevention and cure if contracted. It will educate people on the factors that affect the duration of stay of a patient in the hospital and also help other researchers who seek to know the causes and prevention of typhoid fever.

\subsection{Scope of the Study}

In this work, bishop Shanahan hospital is used as an area of study. The hospital is situated Nsukka urban area and there are other private hospitals which patients also attend. This study examines reported cases of typhoid fever at the Bishop Shanahan Hospital for a period of four years, from January 2012 to December 2016 with names of the patients, age, gender, the duration of stayed by patient in the hospital, the type of typhoid the patient suffered from and the weights of the patient recorded.

\subsection{Aim and Objectives of the Research}

The aim of this study is to determine the relationship between duration of stay, gender, age, type of typhoid, and the weights of typhoid patients.

1) To determine if the duration of stay of typhoid fever patient is dependent on the gender, of the patient;

2) To determine if the duration of stay of a typhoid fever patient is dependent on the age of the patient;

3) To determine if the duration of stay of a typhoid fever patient is dependent on the type of typhoid the patient suffered from;

\section{Literature Review}

Several research works have been done on typhoid fever both in its prevalence in different environment, eradication and control. It is a fact that typhoid fever is an endemic disease that affects underdeveloped and developing countries. Below are some research works that have been carried out on typhoid fever.

A simple linear regression analysis was used to determine if there was a significant relationship between increasing age trend and number of typhoid cases in a blood samples collected from patients suspected to have enteric fever. Samples were also collected from typhoid reporting areas for $\mathrm{H}_{2} \mathrm{~S}$ test to check the presence or absence of sulphur producing bacteria. [8]

Typhoid outbreaks were caused by inadequate disposal of sewage in urban areas thereby contaminating the water supply. [9]

According to [10] studied the burden of typhoid fever in Low-Income and Middle-Income Countries (LMICs) using blood-culture-confirmed incidence rates which applied to the 2010 population after correcting for water-related risks, surveillance, limitations of diagnostic tests, they were able to estimate the typhoid disease burden in Low-Income and Middle-Income Countries (LMICs). They observed that the risk-adjusted typhoid fever burden estimate was more conservative than previous estimates.

Two hundred and seventy-one blood samples from consecutive adults with febrile illness from the University of Benin Teaching Hospital were tested using the widal agglutination test, blood culture and malaria parasite test to determine the preferable option for diagnosis of typhoid fever. [11]

Typhoid fever in China was caused by Salmonella Paratyphi A through the study of the bacteriology, epidemiology, management and antibiotic susceptibility, diagnosis and vaccine development. [12]

Chi-square test, conditional logistic regression and student's t test were used to model the risk factors for typhoid fever among patients who are greater than or equal to 15 years $(\geq 15)$ in Diyarbakir Turkey who have not travelled out from the city for the period of the research. Their main finding of the study was that consumption of vegetables has a significant risk factor for typhoid fever in the area. [13]

\section{Data Collection}

The data for this work are secondary data was collected from in-patients record at bishop Shanahan hospital, Nsukka, Enugu State. The data consist of age, gender of typhoid fever patients in the hospital, their duration of stay, the type of typhoid the patient suffered from before being discharged and the doctor that attended to the patient.

\subsection{Presentation of Data}

The data is arranged in cross-tabs classification. Table 1 is the contingency table for the duration of stay and gender of typhoid patients. Table 2 is the contingency table for the duration of stay and the type of typhoid. Table 3 is the contingency table for the duration of stay and the age of typhoid patients. Table 4 is the contingency table for the duration of stay and the type of doctor that attended to the patient. 
Table 1. Contingency table of duration of stay and gender of typhoid patients.

\begin{tabular}{llll}
\hline & Male & Female & Total \\
\hline Normal & 69 & 102 & 171 \\
Mild & 8 & 30 & 38 \\
Severe & 2 & 6 & 8 \\
Total & 79 & 138 & 217 \\
\hline
\end{tabular}

Table 2. Contingency table of duration of stay and type of typhoid.

\begin{tabular}{llll}
\hline & S. Typhi & P. Typhi & Total \\
\hline Normal & 70 & 101 & 171 \\
Mild & 31 & 7 & 38 \\
Severe & 7 & 1 & 38 \\
Total & 108 & 109 & 217 \\
\hline
\end{tabular}

Table 3. Contingency table of duration of stay and age of typhoid patients.

\begin{tabular}{lllllll}
\hline & Age & & & & & \multirow{2}{*}{ Total } \\
\cline { 2 - 6 } & $\mathbf{1 - 1 8}$ & $\mathbf{1 9 - 3 6}$ & $\mathbf{3 7 - 5 4}$ & $\mathbf{5 5 - 7 2}$ & $\mathbf{7 3 - a b o v e}$ & \\
\hline Normal & 55 & 86 & 22 & 7 & 1 & 171 \\
Mild & 9 & 6 & 2 & 3 & 18 & 38 \\
Severe & 0 & 2 & 0 & 0 & 6 & 8 \\
Total & 64 & 94 & 24 & 10 & 25 & 217 \\
\hline
\end{tabular}

The duration of stay is categorized in three levels: Normal (1-7days), Mild (8-14 days), Severe (15 days and above). Gender is categorized in two levels: Male and female.

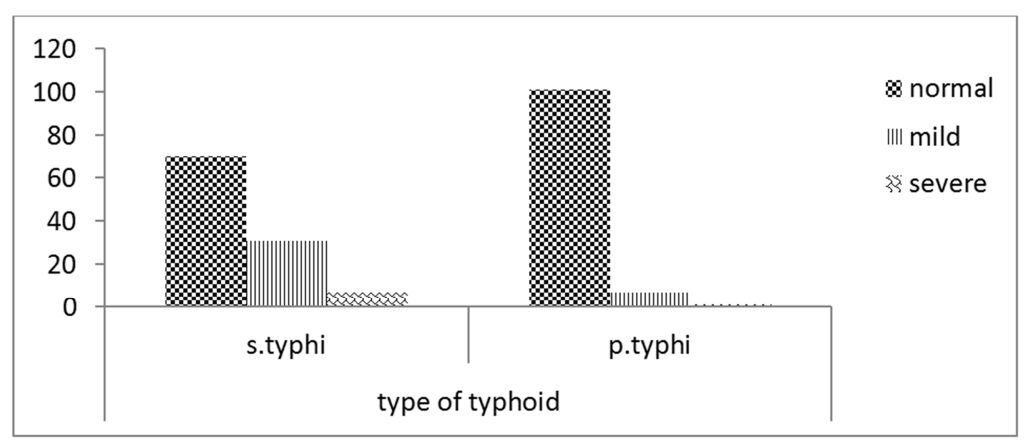

Figure 1. The chart of duration of stay and type of typhoid.

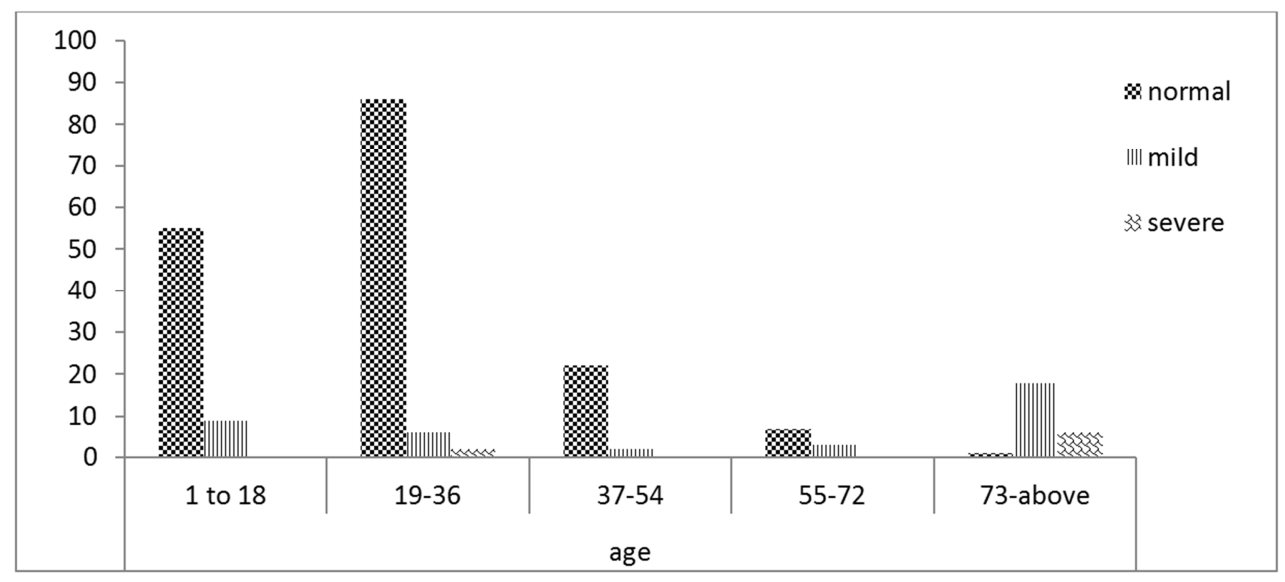

Figure 2. The chart of duration of stay and age of typhoid patients.

\subsection{Chi-square Test}

Given that $Y_{1}, Y_{2}, \ldots, Y_{n}$ forms a sequence of $\mathrm{n}$ independent random variables such that each

$Y_{i} \sim N(0,1)$, we defined the Chi-square variables with $\mathrm{n}$ degrees of freedom as $M=Y_{1}^{2}, Y_{2}^{2}, \ldots, Y_{n}^{2}$ that is $M \sim \chi_{n}^{2}$, where $\chi_{n}^{2}$ denotes the Chi-square with $\mathrm{n}$ degrees of freedom.

The Chi-square test statistic is stated as follows:

$$
X_{c a l}^{2}=\frac{\sum_{i=1}^{r} \sum_{j=1}^{c}\left(O_{i j}-E_{i j}\right)^{2}}{E_{i j}}-\sim X^{2}(r-1)(c-1)
$$

$$
E_{i j}=\frac{r_{i} c_{j}}{n}
$$

Where $o_{i j}$ are the observed frequencies, $e_{i j}$ are the expected frequencies, $r_{i}$ is the row total, $c_{j}$ is the column total and $\mathrm{n}$ is the total number of observations. Chi-square test will be used in this research work to examine if the duration of stay of typhoid fever patient at the above mentioned hospital depends on the following factors, age, gender, type of typhoid and lastly the particular doctor that attended to the patient.

\subsection{The Contingency Table}

In this section, we consider the analysis of data in which the dependent variable and the independent variables are 
categorized, that is, they are measured on normal or ordinal scales. Each scale may have more than two categories. These observations consist of counts or frequencies in cells of a contingency table formed by the cross classification of response variables.

Table 4. The contingency table for a Chi-square Test.

\begin{tabular}{lllll}
\hline & $\mathbf{C}_{\mathbf{i} 1}$ & $\mathbf{C}_{\mathrm{i} 2}$ & $\mathbf{C}_{\mathbf{i j}}$ & Row total \\
\hline $\mathrm{R}_{1}$ & $\mathrm{n}_{11}$ & $\mathrm{n}_{12}$ & $\mathrm{n}_{1 \mathrm{j}}$ & $\mathrm{n}_{1}$ \\
$\mathrm{R}_{2}$ & $\mathrm{n}_{21}$ & $\mathrm{n}_{22}$ & $\mathrm{n}_{2 \mathrm{j}}$ & $\mathrm{n}_{2}$ \\
$\cdot$ & $\cdot$ & $\cdot$ & $\cdot$ & $\cdot$ \\
$\cdot$ & $\cdot$ & $\cdot$ & $\cdot$ & $\cdot$ \\
$\cdot$ & $\cdot$ & $\cdot$ & $\cdot$ & $\cdot$ \\
$\mathrm{R}_{\mathrm{i}}$ & $\mathrm{n}_{\mathrm{i} 1}$ & $\mathrm{n}_{\mathrm{i} 2}$ & $\mathrm{n}_{\mathrm{ij}}$ & $\mathrm{n}_{\mathrm{i}}$ \\
Column total & $\mathrm{n}_{1}$ & $\mathrm{n}_{2}$ & $\mathrm{n}_{\mathrm{j}}$ & $\mathrm{N}$ \\
\hline
\end{tabular}

Where $n_{i j}$ is the frequency associated with the $i^{\text {th }}$ row and $j^{\text {th }}$ column $n_{i}=$ total $i^{\text {th }}$ row, $n_{j}=$ total $j^{\text {th }}$ column and $n$ is the grand total.

\subsection{Assumptions of the Chi-square Test}

Some basic assumptions of the chi-square test are:

i. Each observation has the same probability of being classified into row $\mathrm{i}$ and column $\mathrm{j}$ independent of the other observations.

ii. The two variables should be measured at an ordinal or nominal level, that is, categorical data.

\subsection{Data Analysis}

To test if the duration of stay of typhoid patients is dependent on their gender

Table 5. The Cross-tabulation of duration and gender of typhoid fever patients.

\begin{tabular}{llll}
\hline & Male & Female & Total \\
\hline Normal & $69(62.3)$ & $102(108.7)$ & 171 \\
Mild & $8(13.8)$ & $30(24.2)$ & 38 \\
Severe & $2(2.9)$ & $6(5.1)$ & 8 \\
Total & 79 & 138 & 217 \\
\hline
\end{tabular}

Hypothesis

$\mathrm{H}_{0}=$ The duration of stay of typhoid fever patients is independent of their gender

$\mathrm{H}_{1}=$ The duration of stay of typhoid fever patients is dependent on their gender

$\alpha=0.05$

Test statistic:
$X_{c a l}^{2}=\frac{\sum_{i=1}^{r} \sum_{j=1}^{c}\left(O_{i j}-E_{i j}\right)^{2}}{E_{i j}}-\sim X^{2}(r-1)(c-1)$

Decision rule: reject the null hypothesis $\left(\mathrm{H}_{0}\right)$ if $\chi^{2}$ cal $>$ $X_{\text {tab }}^{2}$ accept if otherwise.

Conclusion: conclude based on the result obtained. This test will be carried out using SPSS.

Table 6. Summary of the chi-square table.

\begin{tabular}{llll}
\hline & Value & df & Asymp. Sig. (2-sided) \\
\hline Pearson Chi-Square & 5.468 & 2 & .065 \\
Likelihood Ratio & 5.822 & 2 & .054 \\
Linear-by-Linear Association & 4.459 & 1 & .035 \\
N of Valid Cases & 217 & & \\
\hline
\end{tabular}

Since $X_{\text {cal }}^{2}=5.468<X_{\text {tab }}^{2}=5.99$, we do not reject $\mathrm{H}_{0}$ and conclude that the duration of stay of typhoid fever patients in the hospital is independent of their gender.

To examine if the duration of stay of typhoid fever patients is dependent on the type of typhoid they suffered from.

Table 7. The cross-tabulation of duration of stay and type of typhoid suffered by the patients

\begin{tabular}{llll}
\hline & S. Typhi & P. Typhi & Total \\
\hline Normal & $70(85.1)$ & $101(85.9)$ & 171 \\
Mild & $31(18.9)$ & $7(19.1)$ & 38 \\
Severe & $7(4.0)$ & $1(4.0)$ & 8 \\
Total & 108 & 109 & 217 \\
\hline
\end{tabular}

$\mathrm{H}_{0}=$ The duration of stay of typhoid fever patients in the hospital is independent of the type of

Typhoid the patient is suffering from.

$\mathrm{H}_{1}=$ The duration of stay of typhoid fever patients in the hospital is dependent on the type of

Typhoid the patient is suffering from.

$\alpha=0.05$

Test statistic:

$$
X_{c a l}^{2}=\frac{\sum_{i=1}^{r} \sum_{j=1}^{c}\left(O_{i j}-E_{i j}\right)^{2}}{E_{i j}}-\sim X^{2}(r-1)(c-1)
$$

Decision rule: reject $\mathrm{H}_{0}$ if $\mathcal{X}^{2}$ cal $>X^{2}$ tab accept if otherwise.

Conclusion: conclude based on the result obtained. This test will be carried out using SPSS.

Table 8. The summary of the chi-square table

\begin{tabular}{llll}
\hline & Value & df & Asymp. Sig. (2 sided) \\
\hline Pearson Chi-Square & 25.274 & 2 & .000 \\
Likelihood Ratio & 27.081 & 2 & .000 \\
Linear-by-Linear Association & 23.124 & 1 & .000 \\
N of Valid Cases & 217 & & \\
\hline
\end{tabular}

Since $X^{2}{ }_{\text {cal }}=25.274>X_{\text {tab }}^{2}=5.99$, we reject $\mathrm{H}_{0}$ and conclude that the duration of stay of typhoid fever patients in the hospital is dependent on the type of typhoid.

To test if the duration of stay of typhoid fever patients in 
the hospital is dependent on age.

Table 9. The cross-tabulation of duration of stay and age of typhoid patients.

\begin{tabular}{|c|c|c|c|c|c|c|c|c|}
\hline & & & \multicolumn{5}{|l|}{ Age } & \multirow{2}{*}{ Total } \\
\hline & & & $1-18$ & $19-36$ & $37-54$ & $55-72$ & 73-above & \\
\hline \multirow{6}{*}{ Duration } & \multirow{2}{*}{ normal } & Count & 55 & 86 & 22 & 7 & 1 & 171 \\
\hline & & Expected Count & 50.4 & 74.1 & 18.9 & 7.9 & 19.7 & 171.0 \\
\hline & \multirow{2}{*}{ mild } & Count & 9 & 6 & 2 & 3 & 18 & 38 \\
\hline & & Expected Count & 11.2 & 16.5 & 4.2 & 1.8 & 4.4 & 38.0 \\
\hline & \multirow{2}{*}{ severe } & Count & 0 & 2 & 0 & 0 & 6 & 8 \\
\hline & & Expected Count & 2.4 & 3.5 & .9 & .4 & .9 & 8.0 \\
\hline \multirow{2}{*}{ Total } & & Count & 64 & 94 & 24 & 10 & 25 & 217 \\
\hline & & Expected Count & 64.0 & 94.0 & 24.0 & 10.0 & 25.0 & 217.0 \\
\hline
\end{tabular}

$\mathrm{H}_{0}$ : The duration of stay of typhoid fever patients is independent of their age.

$\mathrm{H}_{1}$ : The duration of stay of typhoid fever patients is dependent on their age.

$$
\chi_{c a l}^{2}=\frac{\sum_{i=1}^{r} \sum_{j=1}^{c}\left(O_{i j}-E_{i j}\right)^{2}}{E_{i j}}-\sim X^{2}(r-1)(c-1)
$$

$\alpha=0.05$

Test statistic:

Decision rule: reject $\mathrm{H}_{0}$ if $\mathcal{X}_{\text {cal }}^{2}>\mathcal{X}^{2}$ tab accept if otherwise.

Conclusion: conclude based on the result obtained.

Table 10. The summary of the chi-square table.

\begin{tabular}{llll}
\hline & Value & Df & Asymp. Sig. (2-sided) \\
\hline Pearson Chi-Square & 104.416 & 8 & .000 \\
Likelihood Ratio & 89.626 & 8 & .000 \\
Linear-by-Linear Association & 63.884 & 1 & .000 \\
N of Valid Cases & 217 & & \\
\hline
\end{tabular}

Conclusion: Since $X^{2}{ }_{\mathrm{cal}}=104.416>X_{\mathrm{tab}}^{2}=15.51$, we reject $\mathrm{H}_{0}$ and conclude that the duration of stay of typhoid fever patients in the hospital depends on the age of the typhoid fever patient.

\section{Conclusion}

Typhoid fever is a disease that affects underdeveloped and developing countries. Its transmission, prevention and the cure were exhaustively discussed in this work. Data was collected from bishop Shanahan hospital Nsukka, Enugu State. The data was presented according to duration and gender, duration and type of typhoid, duration and age which were put in a tabular form. Analysis was carried out on the data and the methodology used for the analysis is chi-square. The main finding is that duration of stay is dependent on the age of typhoid fever patients and the type of typhoid they suffered from but independent of gender of the typhoid fever patients and the doctor that attended to the patient. This implies that anybody can suffer from typhoid fever irrespective of their gender and also, age can determine how long a typhoid fever patient stays in the hospital. From figure 2 , it can be seen that people between the age brackets of 1936 are prone to typhoid fever than other age brackets but people who are 73years of age and above stay longer in the hospital. This might be as a result of weak immunity due to old age.

\section{References}

[1] Parry, C. M., and Threlfall, E. J. (2008). Antimicrobial resistance in typhoidal and nontyphoidal salmonellae. Current opinion in infectious diseases, 21 (5), 531-538.

[2] Parry, C. M., Hien, T. T., Dougan G., White, N. J., Farrar J. J., and Phil D. (2002). Typhoid fever. The New England Journal of Medicine, 347 (22) 1770-1782.

[3] Maskalyk, J. (2003). Typhoid fever. Canadian Medical Association Journal, 169 (2) 132.

[4] Lin FY, Ho VA, Bay PV, et al (2000). The epidemiology of typhoid fever in the Dong Thap Province, Mekong Delta region of Vietnam. Am J Trop Med Hyg 2000; 62: 644-648.

[5] Osler W (1912). The principles and practice of medicine: designed for the use of practitioners and students of medicine. 8th ed. New York: D. Appleton, 1912: 1-46.

[6] Capoor, M. R., and Nair, D. (2010). Quinolone and Cephalosporin Resistance in Enteric Fever. Journal of Global Infectious Diseases, 2 (3) 258-262. http://doi.org/10.4103/0974$777 X .68529$.

[7] Disease Program at Typhoid Fever, ID-FS-ENT-24 February 2016999 Balmoral Street, Thunder Bay, Health Unit.

[8] Sharma, J., and Malakar, M. (2013). Distribution of Typhoid fever in different rural and urban areas of Lakhimpur District of Assam. International journal of research and development of health, 1 (3) 109-114. 
[9] Khan, K. H., Ganjewala, D., and Rao, K. B. (2008). Recent advancement in typhoid research-a review. Advanced Biotech, 7 (4) $35-40$.

[10] Mogasale, V., Maskery, B., Ochiai, R. L., Lee, J. S., Mogasale, V., Ramani, E., and Wierzba, T. F. (2014). Burden of typhoid fever in low-income and middle-income countries: a systematic, literature-based update with risk-factor adjustment. The Lancet Global health, 2 (10) 570-580.

[11] Enabulele, O., and Awunor, S. N. (2016). Typhoid fever in a Tertiary Hospital in Nigeria: Another look at the Widal agglutination test as a preferred option for diagnosis. Nigerian medical journal: journal of the Nigeria Medical Association, 57 (3) 145-149.

[12] Teh, C. S. J., Chua, K. H., and Thong, K. L. (2014). Paratyphoid fever: splicing the global analyses. International journal of medical sciences, 11 (7) 732-741.

[13] Hosoglu, S., Celen, M. K., Geyik, M. F., Akalin, S., Ayaz, C., Acemoglu, H., and Loeb, (2006). Risk factors for typhoid fever among adult patients in Diyarbakir, Turkey. Epidemiology \& Infection, 134 (3), 612-616. 